\title{
ANHARMONIC EFFECTS IN THE ELECTRON SPIN-LATTICE RELAXATION OF RADIATION DEFECTS IN POTASSIUM SELENATE AND ROCHELLE SALT SINGLE CRYSTALS
}

\author{
V.A. Ivanshin, I.N. KURKIN \\ Kazan State University, Faculty of Physics \\ Lenina 18, 420008 Kazan, Russia \\ W. BRunNer and G. VöLKel \\ Universität Leipzig, Fachbereich Physik \\ Linnéstr. 5, O-7010 Leipzig, Germany \\ (Received July 10, 1991;in final form January 17, 1992)

\begin{abstract}
An anharmonic character for the Raman processes of the electron spin-lattice relaxation of paramagnetic defect centres in $\gamma$-irradiated potassium selenate and Rochelle salt single crystals was observed at low temperatures. PACS numbers: 76.30.Rn, 61.80.Ed, 63.20.Mt, 77.80.-e
\end{abstract}

\section{Introduction}

The spin-lattice relaxation method is rather informative in investigations of peculiarities of vibrational spectra in crystals alongside with such methods as submillimeter spectroscopy, Raman spectroscopy and infrared light absorption [1]. We have measured the spin-lattice relaxation time $T_{1}$ of paramagnetic radicals $\left(\mathrm{SeO}_{3}\right)^{-}$being formed as a result of $\gamma$-irradiation in a potassium selenate $\left(\mathrm{K}_{2} \mathrm{SeO}_{4}\right)$ single crystal which has been grown at the University of Saarbrücken (Germany). As it is known [2], the $\gamma$-irradiation of $\mathrm{K}_{2} \mathrm{SeO}_{4}$ leads to formation of few different radical types. We have studied the radical $\left(\mathrm{SeO}_{3}\right)^{-}$which causes the most intensive EPR line. The dose of irradiation was up to $5 \mathrm{Mrad}$. Measurements have been carried out in the temperature range between $4-180 \mathrm{~K}$ at the frequency of 9.4 $\mathrm{GHz}$ by means of the electron spin echo method at Leipzig University and using pulse saturation method at Kazan University.

The experimental data are compared with those we obtained recently [3] in Rochelle salt with the chemical composition $\mathrm{NaKC}_{4} \mathrm{H}_{4} \mathrm{O}_{6} \cdot 4 \mathrm{H}_{2} \mathrm{O}$. The study 
of the spin-lattice relaxation time $T_{1}$ of paramagnetic centres being formed as a result of X-irradiation has been carried out at two different frequencies 9.4 and $24 \mathrm{GHz}$ in the temperature range between $1.5-300 \mathrm{~K}$. The X-irradiation of Rochelle salt leads to the formation of three different radical types [4]. The $\mathrm{OOC}-\mathrm{C}(\mathrm{OH})-\mathrm{CH}(\mathrm{OH})-\mathrm{COO}$ radical which gives the most intensive EPR line immediately after the irradiation has been studied. Rochelle salt single crystals were irradiated at room temperature using an X-ray tube working at $55 \mathrm{kV}$ and $20 \mathrm{~mA}$. The duration of irradiation was changed from 1 to $5 \mathrm{~h}$ resulting in an increase of the concentration of paramagnetic centres by a factor of three or four up to $10^{19} \mathrm{~cm}^{-3}$. However, a concentration dependence of $T_{1}$ was not observed.

\section{Experimental results}

The results of the $T_{1}$ measurements are presented in Fig. 1 for Rochelle salt and in Fig. 2 for $\mathrm{K}_{2} \mathrm{SeO}_{4}$. It has turned out that the temperature dependence of

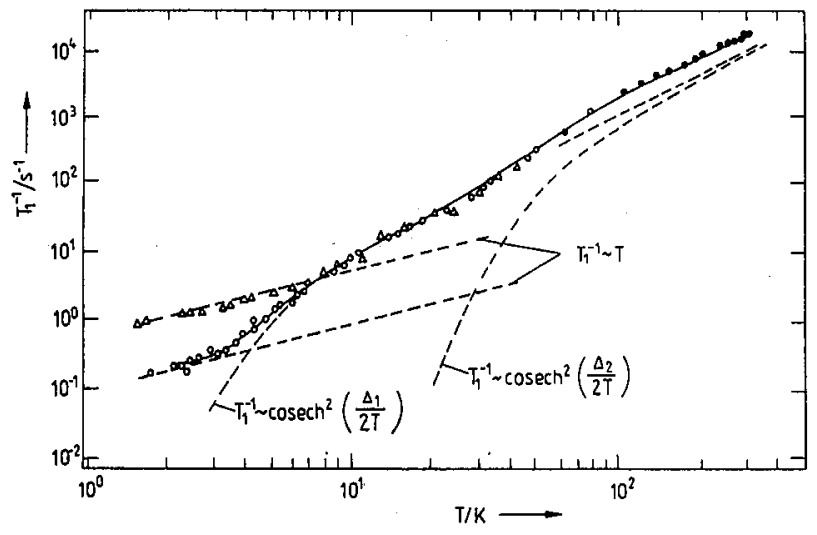

Fig. 1. Temperature dependence of the relaxation rate $T_{1}^{-1}$ in X-irradiated Rochelle salt single crystals measured at $9.5 \mathrm{GHz}(0)$ and $24 \mathrm{GHz}(\Delta) ;(\bullet)$ are taken from [5] (figure taken from Ref. [3]).

$T_{1}^{-1}$ is analogous in both cases and can be described in the whole temperature range of measurements by

$$
T_{1}^{-1}=A T+B_{1} \operatorname{cosech}^{2}\left(\Delta_{1} / 2 T\right)+B_{2} \operatorname{cosech}^{2}\left(\Delta_{2} / 2 T\right),
$$

where the parameters $A, B_{1}, B_{2}, \Delta_{1}, \Delta_{2}$ are given in Table I. The error of measurements leads to an error of the parameters $\Delta_{1}$ and $\Delta_{2}$ of about $10 \%$. 


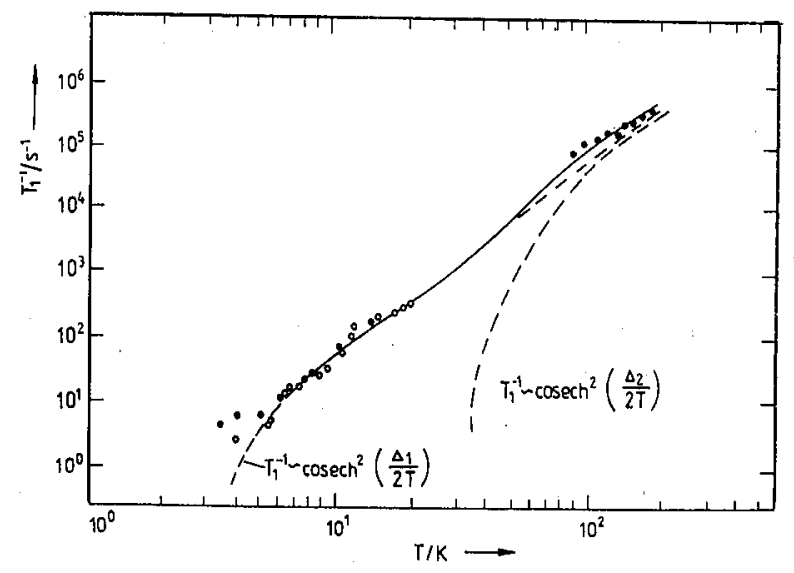

Fig. 2. Temperature dependence of the relaxation rate $T_{1}^{-1}$ in $\gamma$-irradiated $\mathrm{K}_{2} \mathrm{SeO}_{4}$ single crystals measured at $9.4 \mathrm{GHz}$ in Kazan (०) and Leipzig (•).

TABLE I

Parameters of Eq. (1) obtained from a fit with the experimental data shown in Figs. 1 and 2.

\begin{tabular}{c|c|c|c|c|c|c}
\hline \hline Crystal & $\begin{array}{c}\nu \\
{[\mathrm{GHz}]}\end{array}$ & $\begin{array}{c}A \\
{\left[\mathrm{~s}^{-1} \mathrm{~K}^{-1}\right]}\end{array}$ & $\begin{array}{c}B_{1} \\
{\left[\mathrm{~s}^{-1}\right]}\end{array}$ & $\begin{array}{c}B_{2} \\
{\left[\mathrm{~s}^{-1}\right]}\end{array}$ & $\begin{array}{c}\Delta_{1} \\
{[\mathrm{~K}]}\end{array}$ & $\begin{array}{c}\Delta_{2} \\
{[\mathrm{~K}]}\end{array}$ \\
\hline \multirow{2}{*}{ Rochelle salt } & 9.4 & 0.09 & & & & \\
& 24 & 0.5 & 7.4 & 745 & 17.5 & 187 \\
\hline $\mathrm{K}_{2} \mathrm{SeO}_{4}$ & 9.4 & - & 185 & $1.2 \times 10^{5}$ & 27 & 206
\end{tabular}

\section{Discussion}

The first term in (1) corresponds to the direct relaxation process. The terms of type $\operatorname{cosech}^{2}(\Delta / 2 T)$ are connected either with a two-phonon Raman process with an involvement of collective optical lattice vibrations with the energy $k \Delta$ $[6,7]$ or with a Raman process where a localized Einstein mode with the energy $k \Delta$ and acoustic lattice vibrations are involved [8].

Experimental optical investigations in Rochelle salt by means of the Raman spectroscopy [9] indicated the presence of optical lattice vibrations at the wave numbers $128 \mathrm{~cm}^{-1}$ and $135 \mathrm{~cm}^{-1}$. Therefore, the lattice excitation with the wave number $\bar{\nu}_{2}=\Delta_{2} k / h c=(130+5) \mathrm{cm}^{-1}$ determined from the temperature dependence of $T_{1}$ may be attributed to this optical lattice vibration.

As light scattering experiments at optical phonons in $\mathrm{K}_{2} \mathrm{SeO}_{4}$ have revealed there exists indeed a mode at $140 \mathrm{~cm}^{-1}[10]$. This is very close to the wave number $\bar{\nu}_{2}=\Delta_{2} k / h c=143 \mathrm{~cm}^{-1}$ corresponding to $\Delta_{2}$ in potassium selenate.

We can conclude that the relaxation described by the third term in for- 
mula (1) is determined by a Raman process with an involvement of optical lattice vibrations.

The lattice excitations with the wave number $\bar{\nu}_{1}=\Delta_{1} k / h c$, on the other hand, are not so easy to understand. The optical Raman spectra show no vibrations at the corresponding wave numbers $12 \mathrm{~cm}^{-1}$ in Rochelle salt and $18.6 \mathrm{~cm}^{-1}$ in $\mathrm{K}_{2} \mathrm{SeO}_{4}$. The term $\operatorname{cosech}^{2}\left(\Delta_{1} / 2 T\right)$ could be interpreted as caused by a localized Einstein mode at $\Delta_{1}$.

But there is also another interpretation for this term if we take into consideration what frequency the soft mode has in both crystals at very low temperatures. Below $20 \mathrm{~K}$ the soft mode in Rochelle salt and $\mathrm{K}_{2} \mathrm{SeO}_{4}$ takes the value $\bar{\nu}_{\mathrm{SM}}=22 \mathrm{~cm}^{-1}$ [11] and $36 \mathrm{~cm}^{-1}$ [12], respectively. In both cases it becomes apparent that $\bar{\nu}_{1} \simeq \bar{\nu}_{\mathrm{SM}} / 2$. It has been shown theoretically [13] that in a special case of a Raman relaxation process where strongly anharmonic quasi-localized vibrations with frequency $\omega_{\text {local }}$ are involved the subharmonic $\omega_{\text {local }} / 2$ appears in the argument of the Raman term. From this point of view the term $\Delta_{1}$ may be related with an anharmonic quasi-localized vibration at the frozen-in soft-mode frequency.

\section{Conclusion}

According to this interpretation the anharmonic character of the Raman spin-lattice relaxation in irradiated $\mathrm{K}_{2} \mathrm{SeO}_{4}$ and Rochelle salt single crystals points to a distortion of the frozen-in soft mode at temperatures below $20 \mathrm{~K}$ due to the studied paramagnetic defects.

The authors appreciate the experimental assistance of K.P. Chernov and L.L. Sedov in Kazan and are grateful to Dr. J. Albers and Dr. A. Klöpperpieper from the University of Saarbrücken for delivering the crystal.

\section{References}

[1] A.A. Maradudin, Defects and the Vibrational Spectrum of Crystals, Mir, Moscow 1968 (in Russian), p. 353.

[2] K. Aiki, J. Phys. Soc. Jpn. 29, 379 (1970).

[3] V.A. Ivanshin, I.N. Kurkin, G. Völkel, Phys. Status Solidi B 148, K61 (1988).

[4] G.C. Moulton, W.G. Moulton, J. Chem. Phys. 35, 208 (1961).

[5] G. Völkel, H.-E. Müller, W. Flohrer, Phys. Status Solodi B 108, 501 (1981).

[6] C.-Y. Huang, Phys. Rev. 154, 215 (1967).

[7] N. Kumar, K.P. Shina, Physica 34, 387 (1967).

[8] F.F. Popescu, V.V. Grecu, J. Phys. C 15, 1531 (1982).

[9] V. Winterfeldt, Phys. Status Solidi B 100, 235 (1980).

[10] M. Wada, A. Sawada, Y. Ishibashi, Y. Takagi, J. Phys. Soc. Jpn. 42, 1229 (1977).

[11] A.A. Volkov, G.V. Kozlov, E.B. Kryukova, J. Petzelt, Sov. Phys.-JETP 63, 110 (1986).

[12] H.-G. Unruh, W. Eller, G. Kirf, Phys. Status Solidi A 55, 173 (1979).

[13] V.Ya. Zevin, V.I. Konovalov, Sov. Phys.-Solid State 14, 738 (1972). 